\title{
Estimation for the Half Logistic Distribution Based on Double Hybrid Censored Samples
}

\author{
Suk-Bok Kang ${ }^{a}$, Young-Seuk Cho ${ }^{b}$, Jun-Tae Han ${ }^{1, c}$ \\ ${ }^{a}$ Department of Statistics, Yeungnam University \\ ${ }^{b}$ Department of Statistics, Busan National University \\ ${ }^{c}$ National Health Insurance Policy Research Institute
}

\begin{abstract}
Many articles have considered a hybrid censoring scheme, which is a mixture of Type-I and Type-II censoring schemes. We introduce a double hybrid censoring scheme and derive some approximate maximum likelihood estimators(AMLEs) of the scale parameter $\sigma$ for the half logistic distribution under the proposed double hybrid censored samples. The scale parameter is estimated by approximate maximum likelihood estimation method using two different Taylor series expansion types. We also obtain the maximum likelihood estimator(MLE) and the least square estimator(LSE) of the scale parameter $\sigma$ under the proposed double hybrid censored samples. We compare the proposed estimators in the sense of the mean squared error. The simulation procedure is repeated 10,000 times for the sample size $n=20(10) 40$ and various censored samples. The performances of the AMLEs and MLE are very similar in all aspects but the MLE and LSE have not a closed-form expression, some numerical method must be employed.
\end{abstract}

Keywords: Approximate maximum likelihood estimator, double hybrid censored sample, half logistic distribution.

\section{Introduction}

The probability density function(pdf) and the cumulative distribution function(cdf) of the random variable having the half-logistic distribution are given by

$$
f_{X}(x ; \sigma)=\frac{2 \exp \left(-\frac{x}{\sigma}\right)}{\sigma\left\{1+\exp \left(-\frac{x}{\sigma}\right)\right\}^{2}}, \quad x \geq 0, \sigma>0
$$

and

$$
F_{X}(x ; \sigma)=\frac{1-\exp \left(-\frac{x}{\sigma}\right)}{1+\exp \left(-\frac{x}{\sigma}\right)}, \quad x \geq 0, \sigma>0 .
$$

The half logistic distribution obtained by folding the logistic distribution. It can be used as a failure time model in life-testing studies. Balakrishnan (1985) introduced the half logistic distribution as a life testing model with increasing hazard rate. The best linear unbiased estimators(BLUEs) and approximate maximum likelihood estimators(AMLEs) for the location and scale parameters are

\footnotetext{
${ }^{1}$ Corresponding author: Research Fellow, National Health Insurance Policy Research Institute, National Health Insurance Corporation, Seoul 121-749, Korea. E-mail: maru@nhic.or.kr
} 


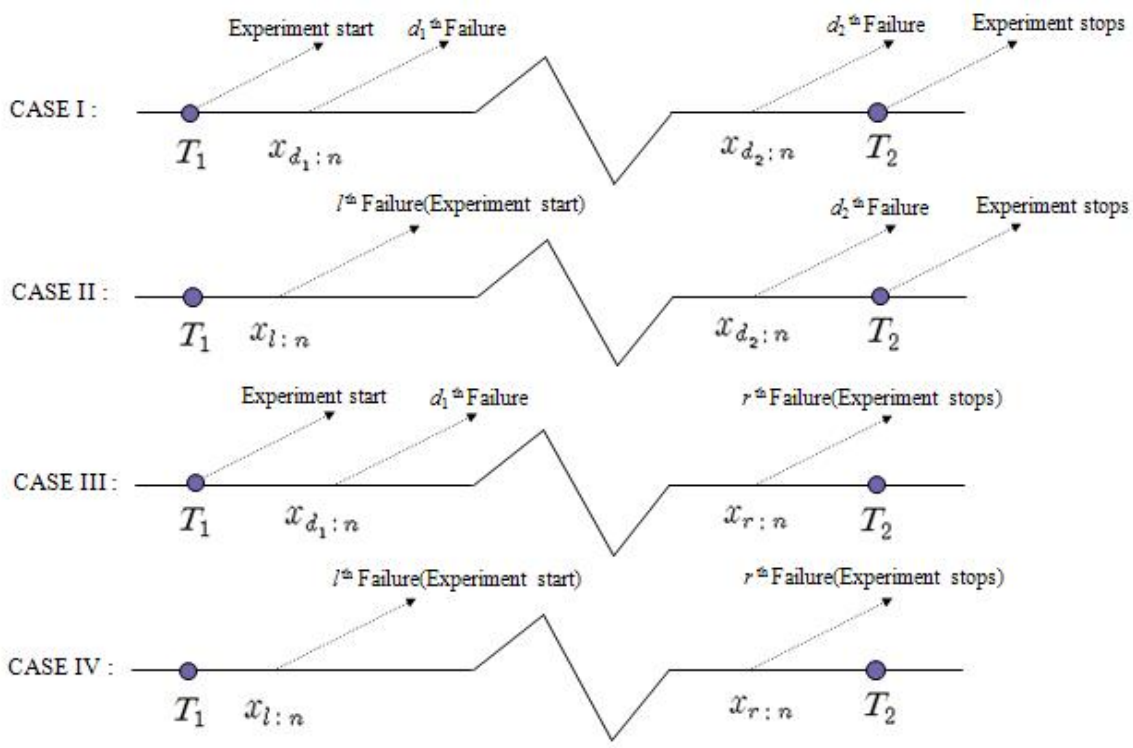

Figure 1: The double hybrid censoring schemes

discussed by Balakrishnan and Puthenpura (1986) and Balakrishnan and Wong (1991). Recently, Han and Kang (2008) derived the AMLEs of the scale parameter and the location parameter in a double Rayleigh distribution based on multiply Type-II censored samples. Kang et al. (2008) derived the AMLEs and the maximum likelihood estimator(MLE) of the scale parameter in a half logistic distribution based on progressive Type-II censored samples.

Hybrid censoring scheme is a mixture of Type-I and Type-II censoring schemes. Let us assume that the ordered lifetimes be denoted by $X_{1: n}, X_{2: n}, \ldots, X_{n: n}$. Epstein (1954) introduced Type-I hybrid censoring scheme, and considered lifetime experiments assuming that the lifetime of each unit follows an exponential distribution. Type-I hybrid censoring scheme in which the life testing experiment is terminated at a random time $T^{*}=\min \left\{X_{r: n}, T\right\}$, where $1 \leq r \leq n$ and $T \in(0, \infty)$ are fixed in advance. As with a conventional Type-I censoring scheme, the termination point here is at most $T$. Childs $e t$ al. (2003) proposed the Type-II hybrid censoring scheme that would terminate the experiment at the random time $T^{*}=\max \left\{X_{r: n}, T\right\}$, where $1 \leq r \leq n$ and $T \in(0, \infty)$ are fixed in advance.

We propose a double hybrid censoring scheme in which the life testing experiment is started at a random time $T_{1}^{*}=\max \left\{X_{l: n}, T_{1}\right\}$ and the life testing experiment is terminated at a random time $T_{2}^{*}=\min \left\{X_{r: n}, T_{2}\right\}$, where $1 \leq l \leq d_{1} \leq d_{2} \leq r \leq n$ and $T_{1}<T_{2}$. A schematic illustration is depicted in Figure 1, when $x_{d_{1}: n}<\cdots<x_{d_{2}: n}$ denote the observed failure times if $x_{d_{1}: n}>T_{1}$ and $x_{d_{2}: n}<T_{2}$ (see, CASE I), and $x_{l: n}<\cdots<x_{d_{2}: n}$ denote the observed failure times if $x_{l: n}>T_{1}$ and $x_{d_{2}: n}<T_{2}$ (see, CASE II), and $x_{d_{1}: n}<\cdots<x_{r: n}$ denote the observed failure times if $x_{d_{1}: n}>T_{1}$ and $x_{r: n}<T_{2}$ (see, CASE III), $x_{l: n}<\cdots<x_{r: n}$ denote the observed failure times if $x_{l: n}>T_{1}$ and $x_{r: n}<T_{2}$ (see, CASE IV).

In this paper, we propose a double hybrid censoring scheme and derive some AMLEs of the scale parameter $\sigma$ for the half logistic distribution under the proposed double hybrid censored samples. The scale parameter is estimated by approximate maximum likelihood estimation method using two different Taylor series expansion types. We also obtain the MLE and the least square estimator(LSE) of the scale parameter $\sigma$ under the proposed double hybrid censored samples. 


\section{Estimation for the Scale Parameter}

We well discuss the maximum likelihood estimation of the scale parameter based on double hybrid censored samples. The log-likelihood function for four different cases follow. Based on the observed data, the log-likelihood function (without the constant term) for Case I is

$$
\ln L_{I}=-S_{I} \ln \sigma+\left(d_{1}-1\right) \ln F\left(T_{1}\right)+\left(n-d_{2}\right) \ln \left\{1-F\left(T_{2}\right)\right\}+\sum_{i=d_{1}}^{d_{2}} \ln f\left(x_{i: n}\right),
$$

for Case II, it is

$$
\ln L_{I I}=-S_{I I} \ln \sigma+(l-1) \ln F\left(x_{l: n}\right)+\left(n-d_{2}\right) \ln \left\{1-F\left(T_{2}\right)\right\}+\sum_{i=l}^{d_{2}} \ln f\left(x_{i: n}\right),
$$

for Case III, it is

$$
\ln L_{I I I}=-S_{I I I} \ln \sigma+\left(d_{1}-1\right) \ln F\left(T_{1}\right)+(n-r) \ln \left\{1-F\left(x_{r: n}\right)\right\}+\sum_{i=d_{1}}^{r} \ln f\left(x_{i: n}\right)
$$

and for Case IV, it is

$$
\ln L_{I V}=-S_{I V} \ln \sigma+(l-1) \ln F\left(x_{l: n}\right)+(n-r) \ln \left\{1-F\left(x_{r: n}\right)\right\}+\sum_{i=l}^{r} \ln f\left(x_{i: n}\right),
$$

where $S_{I}=d_{2}-d_{1}-1, S_{I I}=d_{2}-l-1, S_{I I I}=r-d_{1}-1$ and $S_{I V}=r-l-1$.

The random variable $Z_{i: n}=X_{i: n} / \sigma$ then has a standard half logistic distribution with $p d f$ and $c d f$;

$$
f\left(z_{i: n}\right)=\frac{2 e^{-z_{i: n}}}{\left(1+e^{-z_{i: n}}\right)^{2}}, \quad F\left(z_{i: n}\right)=\frac{1-e^{-z_{i: n}}}{1+e^{-z_{i: n}}} .
$$

The $f\left(z_{i: n}\right), f^{\prime}\left(z_{i: n}\right)$ and $F\left(z_{i: n}\right)$ satisfy as

$$
f^{\prime}\left(z_{i: n}\right)=-F\left(z_{i: n}\right) f\left(z_{i: n}\right), \quad f\left(z_{i: n}\right)=\frac{\left[1-F\left(z_{i: n}\right)\right]\left[1+F\left(z_{i: n}\right)\right]}{2} .
$$

On differentiating the log-likelihood functions with respect to $\sigma$ of $(2.1) \sim(2.4)$ in turn and equation to zero, we obtain the estimating equations as

$$
\begin{aligned}
& \frac{\partial \ln L_{I}}{\partial \sigma}=-\frac{1}{2 \sigma} {\left[2 S_{I}+\left(d_{1}-1\right)\left(\frac{1}{F\left(z_{T_{1}}\right)}-F\left(z_{T_{1}}\right)\right) z_{T_{1}}\right.} \\
&\left.-\left(n-d_{2}\right)\left(1+F\left(z_{T_{2}}\right)\right) z_{T_{2}}-2 \sum_{i=d_{1}}^{d_{2}} F\left(z_{i: n}\right) z_{i: n}\right]=0 \\
& \frac{\partial \ln L_{I I}}{\partial \sigma}=-\frac{1}{2 \sigma}\left[\begin{array}{l}
2 S_{I I}+(l-1)\left(\frac{1}{F\left(z_{l: n}\right)}-F\left(z_{l: n}\right)\right) z_{l: n} \\
\left.-\left(n-d_{2}\right)\left(1+F\left(z_{T_{2}}\right)\right) z_{T_{2}}-2 \sum_{i=l}^{d_{2}} F\left(z_{i: n}\right) z_{i: n}\right]=0
\end{array}\right.
\end{aligned}
$$




$$
\begin{aligned}
\frac{\partial \ln L_{I I I}}{\partial \sigma}=-\frac{1}{2 \sigma}[ & 2 S_{I I I}+\left(d_{1}-1\right)\left(\frac{1}{F\left(z_{T_{1}}\right)}-F\left(z_{T_{1}}\right)\right) z_{T_{1}} \\
& \left.\quad-(n-r)\left(1+F\left(z_{r: n}\right)\right) z_{r: n}-2 \sum_{i=d_{1}}^{r} F\left(z_{i: n}\right) z_{i: n}\right]=0
\end{aligned}
$$

and

$$
\begin{aligned}
\frac{\partial \ln L_{I V}}{\partial \sigma}=-\frac{1}{2 \sigma}[ & 2 S_{I V}+(l-1)\left(\frac{1}{F\left(z_{l: n}\right)}-F\left(z_{l: n}\right)\right) z_{l: n} \\
& \left.\quad(n-r)\left(1+F\left(z_{r: n}\right)\right) z_{r: n}-2 \sum_{i=l}^{r} F\left(z_{i: n}\right) z_{i: n}\right]=0,
\end{aligned}
$$

respectively, where $z_{T_{1}}=T_{1} / \sigma$ and $z_{T_{2}}=T_{2} / \sigma$.

We can find the MLE of $\sigma$ as value $\tilde{\sigma}$ that maximize the log-likelihood functions in (2.1) (2.4) by solving the equations (2.5) (2.8). Since the equations (2.5) (2.8) cannot be solved explicitly, some numerical method must be employed.

Since the log-likelihood equations do not admit explicit solutions, it will be desirable to consider an approximation to the likelihood equations which provide us with explicit estimators for the scale parameter.

We expand the functions $z_{i: n} / F\left(z_{i: n}\right)$ and $F\left(z_{i: n}\right) z_{i: n}$ in Taylor series around the points $\xi_{i}$, where $\xi_{i}=F^{-1}\left(p_{i}\right)=-\ln \left[q_{i} /\left(1+p_{i}\right)\right], p_{i}=i /(n+1), p_{d_{1}^{*}}=\left(p_{d_{1}}+p_{d_{1}-1}\right) / 2, p_{d_{2}^{*}}=\left(p_{d_{2}}+p_{d_{2}+1}\right) / 2$ and $q_{i}=1-p_{i}$.

First, we can approximate the functions by

$$
\begin{aligned}
\frac{z_{i: n}}{F\left(z_{i: n}\right)} & \approx \alpha_{1 i}+\beta_{1 i} z_{i: n}, \\
F\left(z_{i: n}\right) z_{i: n} & \approx \gamma_{1 i}+\delta_{1 i} z_{i: n},
\end{aligned}
$$

where

$$
\begin{aligned}
\alpha_{1 i}=\frac{1-p_{i}^{2}}{2}\left(\frac{\xi_{i}}{p_{i}}\right)^{2}, & \beta_{1 i}=\frac{1}{p_{i}}\left(1-\frac{1-p_{i}^{2}}{2 p_{i}} \xi_{i}\right), \\
\gamma_{1 i}=-\frac{1-p_{i}^{2}}{2} \xi_{i}^{2}, & \delta_{1 i}=\frac{1-p_{i}}{2} \xi_{i}+p_{i} .
\end{aligned}
$$

By substituting the equations (2.9) and (2.10) into the equation (2.5), we may approximate the equation in (2.5) by

$$
\begin{aligned}
& \frac{\partial \ln L_{I}}{\partial \sigma} \simeq-\frac{1}{2 \sigma}\left[2 S_{I}+\left(d_{1}-1\right)\left(\alpha_{1 d_{1}^{*}}+\beta_{1 d_{1}^{*} z_{T_{1}}}\right)-\left(d_{1}-1\right)\left(\gamma_{1 d_{1}^{*}}+\delta_{1 d_{1}^{*} z_{T_{1}}}\right)\right. \\
& \left.-\left(n-d_{2}\right) z_{T_{2}}-\left(n-d_{2}\right)\left(\gamma_{1 d_{2}^{*}}+\delta_{1 d_{2}^{*}} z_{T_{2}}\right)-2 \sum_{i=d_{1}}^{d_{2}}\left(\gamma_{1 i}+\delta_{1 i} z_{i: n}\right)\right] \\
& =0 \text {. }
\end{aligned}
$$


We can derive an estimator of $\sigma$ as follows;

$$
\hat{\sigma}_{1 I}=-\frac{B_{1 I}}{A_{1 I}}
$$

where

$$
\begin{aligned}
& A_{1 I}=2 S_{I}+\left(d_{1}-1\right)\left(\alpha_{1 d_{1}^{*}}-\gamma_{1 d_{1}^{*}}\right)-\left(n-d_{2}\right) \gamma_{1 d_{2}^{*}}-2 \sum_{i=d_{1}}^{d_{2}} \gamma_{1 i}, \\
& B_{1 I}=\left(d_{1}-1\right)\left(\beta_{1 d_{1}^{*}}-\delta_{1 d_{1}^{*}}\right) T_{1}-\left(n-d_{2}\right)\left(1+\delta_{1 d_{2}^{*}}\right) T_{2}-2 \sum_{i=d_{1}}^{d_{2}} \delta_{1 i} X_{i: n} .
\end{aligned}
$$

Since $\alpha_{1 d_{1}^{*}}>0, \gamma_{1 d_{1}^{*}}<0, \beta_{1 d_{1}^{*}}-\delta_{1 d_{1}^{*}}<0$ and $\alpha_{1 i}>0$, the estimator $\hat{\sigma}_{1 I}$ is always positive.

Similarly, for Case II, Case III, and Case IV, the AMLE of $\sigma$ can be obtained by solving

$$
\begin{aligned}
\frac{\partial \ln L_{I I}}{\partial \sigma} \simeq & -\frac{1}{2 \sigma}\left[2 S_{I I}+(l-1)\left(\alpha_{1 l}+\beta_{1 I} z_{l: n}\right)-(l-1)\left(\gamma_{1 l}+\delta_{11 z_{l: n}}\right)\right. \\
& \left.-\left(n-d_{2}\right) z_{T_{2}}-\left(n-d_{2}\right)\left(\gamma_{1 d_{2}^{*}}+\delta_{1 d_{2}^{*} z_{T_{2}}}\right)-2 \sum_{i=l}^{d_{2}}\left(\gamma_{1 i}+\delta_{1 i} z_{i: n}\right)\right] \\
= & 0, \\
\frac{\partial \ln L_{I I I} \simeq}{\partial \sigma} \simeq & -\frac{1}{2 \sigma}\left[2 S_{I I I}+\left(d_{1}-1\right)\left(\alpha_{1 d_{1}^{*}}+\beta_{1 d_{1}^{*} z_{T_{1}}}\right)-\left(d_{1}-1\right)\left(\gamma_{1 d_{1}^{*}}+\delta_{1 d_{1}^{*} z_{T_{1}}}\right)\right. \\
& \left.-(n-r) z_{r: n}-(n-r)\left(\gamma_{1 r}+\delta_{1 r} z_{r: n}\right)-2 \sum_{i=d_{1}}^{r}\left(\gamma_{1 i}+\delta_{1 i} z_{i: n}\right)\right] \\
= & 0
\end{aligned}
$$

and

$$
\begin{aligned}
\frac{\partial \ln L_{I V}}{\partial \sigma} \simeq & -\frac{1}{2 \sigma}\left[2 S_{I V}+(l-1)\left(\alpha_{1 l}+\beta_{11} z_{l: n}\right)-(l-1)\left(\gamma_{1 l}+\delta_{1 l} z_{l: n}\right)\right. \\
& \left.-(n-r) z_{r: n}-(n-r)\left(\gamma_{1 r}+\delta_{1 r} z_{r: n}\right)-2 \sum_{i=l}^{r}\left(\gamma_{1 i}+\delta_{1 i} z_{i: n}\right)\right] \\
= & 0,
\end{aligned}
$$

respectively. Therefore, for Case II, Case III, and Case IV, we obtain the AMLE of $\sigma$ as follows;

$$
\begin{gathered}
\hat{\sigma}_{1 I I}=\frac{-B_{1 I I}}{A_{1 I I}}, \\
\hat{\sigma}_{1 I I I}=\frac{-B_{1 I I I}}{A_{1 I I I}}, \\
\hat{\sigma}_{1 I V}=\frac{-B_{1 I V}}{A_{1 I V}},
\end{gathered}
$$


where

$$
\begin{aligned}
& A_{1 I I}=2 S_{I I}+(l-1)\left(\alpha_{1 l}-\gamma_{1 l}\right)-\left(n-d_{2}\right) \gamma_{1 d_{2}^{*}}-2 \sum_{i=l}^{d_{2}} \gamma_{1 i}, \\
& B_{1 I I}=(l-1)\left(\beta_{1 l}-\delta_{1 l}\right) X_{l: n}-\left(n-d_{2}\right)\left(1+\delta_{1 d_{2}^{*}}\right) T_{2}-2 \sum_{i=l}^{d_{2}} \delta_{1 i} X_{i: n}, \\
& A_{1 I I I}=2 S_{I I I}+\left(d_{1}-1\right)\left(\alpha_{1 d_{1}^{*}}-\gamma_{1 d_{1}^{*}}\right)-(n-r) \gamma_{1 r}-2 \sum_{i=d_{1}}^{r} \gamma_{1 i}, \\
& B_{1 I I I}=\left(d_{1}-1\right)\left(\beta_{1 d_{1}^{*}}-\delta_{1 d_{1}^{*}}\right) z_{T_{1}}-(n-r)\left(1+\delta_{1 r}\right) X_{r: n}-2 \sum_{i=d_{1}}^{r} \delta_{1 i} X_{i: n}, \\
& A_{1 I V}=2 S_{I V}+(l-1)\left(\alpha_{1 l}-\gamma_{1 l}\right)-(n-r) \gamma_{1 r}-2 \sum_{i=l}^{r} \gamma_{1 i}, \\
& B_{1 I V}=(l-1)\left(\beta_{1 l}-\delta_{1 l}\right) X_{l: n}-(n-r)\left(1+\delta_{1 r}\right) X_{r: n}-2 \sum_{i=l}^{r} \delta_{1 i} X_{i: n} .
\end{aligned}
$$

The AMLE $\hat{\sigma}_{1}\left(\hat{\sigma}_{1 I}, \hat{\sigma}_{1 I I}, \hat{\sigma}_{1 I I I}\right.$, and $\left.\hat{\sigma}_{1 I V}\right)$ is always positive in all case.

Second, we can approximate the functions by the equations,

$$
\begin{aligned}
& \frac{1}{F\left(z_{i: n}\right)} \approx \alpha_{2 i}+\beta_{2 i} z_{i: n}, \\
& F\left(z_{i: n}\right) \approx \gamma_{2 i}+\delta_{2 i} z_{i: n},
\end{aligned}
$$

where

$$
\begin{aligned}
\alpha_{2 i} & =\frac{1}{p_{i}}\left[1+\frac{q_{i}}{2 p_{i}} \xi_{i}\right], & \beta_{2 i} & =-\frac{q_{i}}{2 p_{i}}, \\
\gamma_{2 i} & =p_{i}-\frac{1-p_{i}^{2}}{2} \xi_{i}, & \delta_{2 i} & =\frac{1-p_{i}^{2}}{2} .
\end{aligned}
$$

By substituting the equations (2.19) and (2.20) into the equation (2.5), we may approximate the equation in (2.5) by

$$
\begin{aligned}
\frac{\partial \ln L_{I}}{\partial \sigma} \simeq & -\frac{1}{2 \sigma}\left[2 S_{I}+\left(d_{1}-1\right)\left\{\left(\alpha_{2 d_{1}^{*}}+\beta_{2 d_{1}^{*}} z_{T_{1}}\right)-\left(\gamma_{2 d_{1}^{*}}+\delta_{2 d_{1}^{*}} z_{T_{1}}\right) z_{T_{1}}\right.\right. \\
& \left.-\left(n-d_{2}\right) z_{T_{2}}-\left(n-d_{2}\right)\left(\gamma_{2 d_{2}^{*}}+\delta_{2 d_{2}^{*}} z_{T_{2}}\right) z_{T_{2}}-2 \sum_{i=d_{1}}^{d_{2}}\left(\gamma_{2 i}+\delta_{2 i} z_{i: n}\right) z_{i: n}\right] \\
= & 0 .
\end{aligned}
$$

The equation (2.21) is a quadratic equation in $\sigma$, with its roots given by

$$
\hat{\sigma}_{2 I}=\frac{-B_{2 I} \pm \sqrt{B_{2 I}^{2}-8 S_{I} C_{2 I}}}{4 S_{I}},
$$


where

$$
\begin{aligned}
& B_{2 I}=\left(d_{1}-1\right)\left(\alpha_{2 d_{1}^{*}}-\gamma_{2 d_{1}^{*}}\right) T_{1}-\left(n-d_{2}\right)\left(1+\gamma_{2 d_{2}^{*}}\right) T_{2}-2 \sum_{i=d_{1}}^{d_{2}} \gamma_{2 i} X_{i: n}, \\
& C_{2 I}=\left(d_{1}-1\right)\left(\beta_{2 d_{1}^{*}}-\gamma_{2 d_{1}^{*}}\right) T_{1}^{2}-\left(n-d_{2}\right) \delta_{2 d_{2}^{*}} T_{2}^{2}-2 \sum_{i=d_{1}}^{d_{2}} \delta_{2 i} X_{i: n}^{2} .
\end{aligned}
$$

Since $C_{2 I}<0$, only one root is admissible, and hence the AMLE of $\sigma$ is given by

$$
\hat{\sigma}_{2 I}=\frac{-B_{2 I}+\sqrt{B_{2 I}^{2}-8 S_{I} C_{2 I}}}{4 S_{I}} .
$$

Similarly, for Case II, the AMLE of $\sigma$ can be obtained by solving

$$
\begin{aligned}
\frac{\partial \ln L_{I I}}{\partial \sigma} \simeq & -\frac{1}{2 \sigma}\left[2 S_{I I}+(l-1)\left\{\left(\alpha_{2 l}+\beta_{2 l} z_{l: n}\right)-\left(\gamma_{2 l}+\delta_{2 l} z_{l: n}\right)\right\} z_{l: n}\right. \\
& \left.-\left(n-d_{2}\right) z_{T_{2}}-\left(n-d_{2}\right)\left(\gamma_{2 d_{2}^{*}}+\delta_{2 d_{2}^{*}} z_{T_{2}}\right) z_{T_{2}}-2 \sum_{i=l}^{d_{2}}\left(\gamma_{2 i}+\delta_{2 i} z_{i: n}\right) z_{i: n}\right] \\
= & 0 .
\end{aligned}
$$

For Case III, the AMLE of $\sigma$ can be obtained by solving

$$
\begin{aligned}
\frac{\partial \ln L_{I I I}}{\partial \sigma} \simeq & -\frac{1}{2 \sigma}\left[2 S_{I I I}+\left(d_{1}-1\right)\left\{\left(\alpha_{2 d_{1}^{*}}+\beta_{2 d_{1}^{*}} z_{T_{1}}\right)-\left(\gamma_{2 d_{1}^{*}}+\delta_{2 d_{1}^{*}} z_{T_{1}}\right)\right\} z_{T_{1}}\right. \\
& \left.-(n-r) z_{r: n}-(n-r)\left(\gamma_{2 r}+\delta_{2 r} z_{r: n}\right) z_{r: n}-2 \sum_{i=d_{1}}^{r}\left(\gamma_{2 i}+\delta_{2 i} z_{i: n}\right) z_{i: n}\right] \\
= & 0 .
\end{aligned}
$$

For Case IV, the AMLE of $\sigma$ can be obtained by solving

$$
\begin{aligned}
\frac{\partial \ln L_{I V}}{\partial \sigma} \simeq & -\frac{1}{2 \sigma}\left[2 S_{I V}+(l-1)\left\{\left(\alpha_{2 l}+\beta_{2 l} z_{l: n}\right)-\left(\gamma_{2 l}+\delta_{2 l} z_{l: n}\right)\right\} z_{l: n}\right. \\
& \left.-(n-r) z_{r: n}-(n-r)\left(\gamma_{2 r}+\delta_{2 r} z_{r: n}\right) z_{r: n}-2 \sum_{i=l}^{r}\left(\gamma_{2 i}+\delta_{2 i} z_{i: n}\right) z_{i: n}\right] \\
= & 0 .
\end{aligned}
$$

Therefore, for Case II, Case III, and Case IV, we obtain the AMLE of $\sigma$ as follows;

$$
\begin{gathered}
\hat{\sigma}_{2 I I}=\frac{-B_{2 I I}+\sqrt{B_{2 I I}^{2}-8 S_{I} C_{2 I I}}}{4 S_{I I}}, \\
\hat{\sigma}_{2 I I I}=\frac{-B_{2 I I I}+\sqrt{B_{2 I I I}^{2}-8 S_{I} C_{2 I I I}}}{4 S_{I I I}},
\end{gathered}
$$




$$
\hat{\sigma}_{2 I V}=\frac{-B_{2 I V}+\sqrt{B_{2 I V}^{2}-8 S_{I} C_{2 I V}}}{4 S_{I V}},
$$

where

$$
\begin{aligned}
& B_{2 I I}=(l-1)\left(\alpha_{2 l}-\gamma_{2 l}\right) X_{l: n}-\left(n-d_{2}\right)\left(1+\gamma_{2 d_{2}^{*}}\right) T_{2}-2 \sum_{i=l}^{d_{2}} \gamma_{2 i} X_{i: n}, \\
& C_{2 I I}=(l-1)\left(\beta_{2 l}-\gamma_{2 l}\right) X_{l: n}^{2}-\left(n-d_{2}\right) \delta_{2 d_{2}^{*}} T_{2}^{2}-2 \sum_{i=l}^{d_{2}} \delta_{2 i} X_{i: n}^{2}, \\
& B_{2 I I I}=\left(d_{1}-1\right)\left(\alpha_{2 d_{1}^{*}}-\gamma_{2 d_{1}^{*}}\right) z_{T_{1}}-(n-r)\left(1+\gamma_{2 r}\right) X_{r: n}-2 \sum_{i=d_{1}}^{r} \gamma_{2 i} X_{i: n}, \\
& C_{2 I I I}=\left(d_{1}-1\right)\left(\beta_{2 d_{1}^{*}}-\gamma_{2 d_{1}^{*}}\right) z_{T_{1}}^{2}-(n-r) \delta_{2 r} X_{r: n}^{2}-2 \sum_{i=d_{1}}^{r} \delta_{2 i} X_{i: n}^{2}, \\
& B_{2 I V}=(l-1)\left(\alpha_{2 l}-\gamma_{2 l}\right) X_{l: n}-(n-r)\left(1+\gamma_{2 r}\right) X_{r: n}-2 \sum_{i=l}^{r} \gamma_{2 i} X_{i: n}, \\
& C_{2 I V}=(l-1)\left(\beta_{2 l}-\gamma_{2 l}\right) X_{l: n}^{2}-(n-r) \delta_{2 r} X_{r: n}^{2}-2 \sum_{i=l}^{r} \delta_{2 i} X_{i: n}^{2} .
\end{aligned}
$$

The LSE is obtained by minimizing

$$
Q=\sum_{i=i}^{n}\left(F\left(x_{i: n}\right)-\frac{i}{n+1}\right)^{2} .
$$

We can modify the equation (2.30) based on double hybrid censored samples as follows: For Case I, it is

$$
Q_{I}=\sum_{i=d_{1}}^{d_{2}}\left(F\left(x_{i: n}\right)-\frac{i}{n+1}\right)^{2},
$$

for Case II, it is

$$
Q_{I I}=\sum_{i=l}^{d_{2}}\left(F\left(x_{i: n}\right)-\frac{i}{n+1}\right)^{2},
$$

for Case III, it is

$$
Q_{I I I}=\sum_{i=d_{1}}^{r}\left(F\left(x_{i: n}\right)-\frac{i}{n+1}\right)^{2},
$$

and for Case IV, it is

$$
Q_{I V}=\sum_{i=l}^{r}\left(F\left(x_{i: n}\right)-\frac{i}{n+1}\right)^{2} .
$$


On differentiating the equations $(2.31) \sim(2.34)$ with respect to $\sigma$ in turn and the equation to zero. The resulting equations are given below.

$$
\begin{aligned}
& 2 \sum_{i=d_{1}}^{d_{2}}\left(F\left(x_{i: n}\right)-\frac{i}{n+1}\right) f\left(x_{i: n}\right)=0, \\
& 2 \sum_{i=l}^{d_{2}}\left(F\left(x_{i: n}\right)-\frac{i}{n+1}\right) f\left(x_{i: n}\right)=0, \\
& 2 \sum_{i=d_{1}}^{r}\left(F\left(x_{i: n}\right)-\frac{i}{n+1}\right) f\left(x_{i: n}\right)=0, \\
& 2 \sum_{i=l}^{r}\left(F\left(x_{i: n}\right)-\frac{i}{n+1}\right) f\left(x_{i: n}\right)=0 .
\end{aligned}
$$

Since the equations $(2.35) \sim(2.38)$ cannot be solved explicitly, solution of the equations $(2.35) \sim(2.38)$ are obtained by using numerical method.

\section{Simulated Results}

From the above formula, the mean squared errors of the estimators are simulated by Monte Carlo method (based on 10,000 Monte Carlo runs) for sample size $n=20(10) 40$, and different $l, r, T_{1}$ and $T_{2}$ values. We mainly compare the performances of the proposed estimators of the scale parameter $\sigma$, in terms of their biases and mean squared errors(MSEs) for different censoring schemes. The convergence of Newton-Raphson method depended on the choice of the initial values. For this reason, the AMLE $\hat{\sigma}_{1}\left(\hat{\sigma}_{1 I}, \hat{\sigma}_{1 I I}, \hat{\sigma}_{1 I I I}\right.$ and $\left.\hat{\sigma}_{1 I V}\right)$ is used as starting values for the iterations, the MLE $\tilde{\sigma}$ and LSE $\bar{\sigma}$ are obtained using Newton-Raphson method.

From Table A.1 A.2, the following general observations can be made. For all the method, for fixed $l, r, T_{1}$ and $T_{2}$, the MSEs decrease as $n$ increases from 20 to 40 , for fixed $n, l, T_{1}$ and $T_{2}$, the MSEs decrease as $r$ increases.

The performances of the AMLEs and MLE are very similar in all aspects. The AMLE $\hat{\sigma}_{1}$ is a linear function of available order statistics and the performance is good. For $n=20, l=1$ and $r=20$, the LSE $\bar{\sigma}$ is more efficient than the others. 


\section{Appendix:}

Table A.1: The relative mean squared errors and biases for the estimators of the scale parameter $\sigma$

\begin{tabular}{|c|c|c|c|c|c|c|c|c|}
\hline \multirow[b]{2}{*}{$T_{1}$} & \multirow[b]{2}{*}{$T_{2}$} & \multirow[b]{2}{*}{$n$} & \multirow[b]{2}{*}{$l$} & \multirow[b]{2}{*}{$r$} & \multicolumn{4}{|c|}{ MSE(Bias) } \\
\hline & & & & & AMLE $\hat{\sigma}_{1}$ & AMLE $\hat{\sigma}_{2}$ & MLE $\tilde{\sigma}$ & $\operatorname{LSE} \bar{\sigma}$ \\
\hline \multirow[t]{19}{*}{0.3} & \multirow[t]{19}{*}{3.0} & \multirow[t]{7}{*}{20} & 1 & 20 & $0.0394(0.0034)$ & $0.0383(0.0259)$ & $0.0400(0.0085)$ & $0.0354(0.0180)$ \\
\hline & & & 3 & 17 & $0.0417(-0.0057)$ & $0.0491(0.0196)$ & $0.0420(0.0003)$ & $0.0636(0.0750)$ \\
\hline & & & 3 & 16 & $0.0429(-0.0089)$ & $0.0491(0.0174)$ & $0.0433(-0.0027)$ & $0.0629(0.0680)$ \\
\hline & & & 3 & 15 & $0.0449(-0.0108)$ & $0.0503(0.0183)$ & $0.0453(-0.0046)$ & $0.0623(0.0606)$ \\
\hline & & & 4 & 17 & $0.0416(-0.0065)$ & $0.0513(0.0307)$ & $0.0420(0.0003)$ & $0.0644(0.0068)$ \\
\hline & & & 5 & 17 & $0.0405(-0.0096)$ & $0.0563(0.0471)$ & $0.0420(0.0004)$ & $0.0565(-0.0824)$ \\
\hline & & & 6 & 17 & $0.0372(-0.0193)$ & $0.0659(0.0721)$ & $0.0420(0.0003)$ & $0.0477(-0.1487)$ \\
\hline & & \multirow[t]{6}{*}{30} & 1 & 30 & $0.0264(0.0026)$ & $0.0274(0.0160)$ & $0.0267(0.0060)$ & $0.0291(0.0182)$ \\
\hline & & & 3 & 27 & $0.0272(-0.0007)$ & $0.0316(0.0167)$ & $0.0274(0.0029)$ & $0.0587(0.1264)$ \\
\hline & & & 3 & 26 & $0.0276(-0.0026)$ & $0.0317(0.0140)$ & $0.0277(0.0011)$ & $0.0588(0.1241)$ \\
\hline & & & 5 & 27 & $0.0269(-0.0048)$ & $0.0359(0.0390)$ & $0.0274(0.0030)$ & $0.0742(0.0629)$ \\
\hline & & & 6 & 27 & $0.0255(-0.0210)$ & $0.0411(0.0563)$ & $0.0274(0.0030)$ & $0.0674(-0.0216)$ \\
\hline & & & 6 & 25 & $0.0280(-0.0068)$ & $0.0344(0.0371)$ & $0.0282(-0.0011)$ & $0.0657(-0.0303)$ \\
\hline & & \multirow[t]{6}{*}{40} & 1 & 40 & $0.0198(0.0010)$ & $0.0212(0.0104)$ & $0.0199(0.0036)$ & $0.0238(0.0128)$ \\
\hline & & & 3 & 35 & $0.0207(-0.0031)$ & $0.0232(0.0090)$ & $0.0207(-0.0005)$ & $0.0482(0.1259)$ \\
\hline & & & 4 & 37 & $0.0203(-0.0003)$ & $0.0239(0.0205)$ & $0.0203(0.0023)$ & $0.0647(0.1586)$ \\
\hline & & & 5 & 37 & $0.0204(-0.0037)$ & $0.0254(0.0339)$ & $0.0203(0.0023)$ & $0.0771(0.1509)$ \\
\hline & & & 6 & 30 & $0.0226(-0.0078)$ & $0.0259(0.0308)$ & $0.0227(-0.0036)$ & $0.0758(0.0690)$ \\
\hline & & & 11 & 30 & $0.0225(-0.0121)$ & $0.0359(0.0866)$ & $0.0227(-0.0035)$ & $0.0419(-0.1868)$ \\
\hline \multirow[t]{19}{*}{0.3} & \multirow[t]{19}{*}{2.0} & \multirow[t]{7}{*}{20} & 1 & 20 & $0.0509(0.0171)$ & $0.0591(0.0408)$ & $0.0516(0.0224)$ & $0.0363(0.0127)$ \\
\hline & & & 3 & 17 & $0.0513(0.0136)$ & $0.0732(0.0687)$ & $0.0528(0.0202)$ & $0.0623(0.0777)$ \\
\hline & & & 3 & 16 & $0.0530(0.0098)$ & $0.0765(0.0651)$ & $0.0543(0.0161)$ & $0.0629(0.0744)$ \\
\hline & & & 3 & 15 & $0.0546(0.0042)$ & $0.0799(0.0588)$ & $0.0558(0.0104)$ & $0.0629(0.0661)$ \\
\hline & & & 4 & 17 & $0.0507(0.0126)$ & $0.0841(0.0915)$ & $0.0528(0.0202)$ & $0.0660(0.0230)$ \\
\hline & & & 5 & 17 & $0.0508(0.0084)$ & $0.1044(0.1227)$ & $0.0528(0.0203)$ & $0.0599(-0.0626)$ \\
\hline & & & 6 & 17 & $0.0537(-0.0084)$ & $0.1408(0.1664)$ & $0.0528(0.0203)$ & $0.0493(-0.1396)$ \\
\hline & & \multirow[t]{6}{*}{30} & 1 & 30 & $0.0324(0.0105)$ & $0.0368(0.0303)$ & $0.0327(0.0141)$ & $0.0303(0.0151)$ \\
\hline & & & 3 & 27 & $0.0322(0.0098)$ & $0.0408(0.0451)$ & $0.0328(0.0140)$ & $0.0551(0.1097)$ \\
\hline & & & 3 & 26 & $0.0324(0.0096)$ & $0.0410(0.0454)$ & $0.0330(0.0135)$ & $0.0553(0.1105)$ \\
\hline & & & 5 & 27 & $0.0320(0.0085)$ & $0.0479(0.0768)$ & $0.0328(0.0140)$ & $0.0719(0.0652)$ \\
\hline & & & 6 & 27 & $0.0341(0.0032)$ & $0.0544(0.0984)$ & $0.0328(0.0140)$ & $0.0684(-0.0064)$ \\
\hline & & & 6 & 25 & $0.0331(0.0077)$ & $0.0610(0.0937)$ & $0.0336(0.0121)$ & $0.0678(-0.0145)$ \\
\hline & & \multirow[t]{6}{*}{40} & 1 & 40 & $0.0242(0.0067)$ & $0.0272(0.0231)$ & $0.0243(0.0095)$ & $0.0256(0.0128)$ \\
\hline & & & 3 & 35 & $0.0241(0.0064)$ & $0.0289(0.0326)$ & $0.0244(0.0092)$ & $0.0461(0.1128)$ \\
\hline & & & 4 & 37 & $0.0239(0.0060)$ & $0.0305(0.0424)$ & $0.0243(0.0094)$ & $0.0616(0.1414)$ \\
\hline & & & 5 & 37 & $0.0237(0.0056)$ & $0.0323(0.0541)$ & $0.0243(0.0094)$ & $0.0720(0.1325)$ \\
\hline & & & 6 & 30 & $0.0266(0.0009)$ & $0.0448(0.0625)$ & $0.0260(0.0032)$ & $0.0768(0.0752)$ \\
\hline & & & 11 & 30 & $0.0251(-0.0064)$ & $0.0639(0.1173)$ & $0.0260(0.0032)$ & $0.0419(-0.1867)$ \\
\hline
\end{tabular}


Table A.2: The relative mean squared errors and biases for the estimators of the scale parameter $\sigma$

\begin{tabular}{|c|c|c|c|c|c|c|c|c|}
\hline \multirow[b]{2}{*}{$T_{1}$} & \multirow[b]{2}{*}{$T_{2}$} & \multirow[b]{2}{*}{$n$} & \multirow[b]{2}{*}{$l$} & \multirow[b]{2}{*}{$r$} & \multicolumn{4}{|c|}{ MSE(Bias) } \\
\hline & & & & & $\operatorname{AMLE} \hat{\sigma}_{1}$ & AMLE $\hat{\sigma}_{2}$ & MLE $\tilde{\sigma}$ & LSE $\bar{\sigma}$ \\
\hline \multirow[t]{19}{*}{0.4} & 3.0 & 20 & 1 & 20 & $0.0394(0.0037)$ & $0.0391(0.0287)$ & $0.0400(0.0085)$ & $0.0357(0.0218)$ \\
\hline & & & 3 & 17 & $0.0416(-0.0057)$ & $0.0502(0.0227)$ & $0.0420(0.0003)$ & $0.0627(0.0737)$ \\
\hline & & & 3 & 16 & $0.0429(-0.0089)$ & $0.0500(0.0205)$ & $0.0433(-0.0027)$ & $0.0624(0.0685)$ \\
\hline & & & 3 & 15 & $0.0448(-0.0108)$ & $0.0512(0.0215)$ & $0.0453(-0.0046)$ & $0.0620(0.0617)$ \\
\hline & & & 4 & 17 & $0.0416(-0.0066)$ & $0.0529(0.0366)$ & $0.0420(0.0003)$ & $0.0642(0.0118)$ \\
\hline & & & 5 & 17 & $0.0405(-0.0097)$ & $0.0576(0.0537)$ & $0.0420(0.0004)$ & $0.0570(-0.0738)$ \\
\hline & & & 6 & 17 & $0.0372(-0.0192)$ & $0.0669(0.0773)$ & $0.0421(0.0003)$ & $0.0483(-0.1415)$ \\
\hline & & 30 & 1 & 30 & $0.0264(0.0028)$ & $0.0279(0.0163)$ & $0.0267(0.0060)$ & $0.0288(0.0206)$ \\
\hline & & & 3 & 27 & $0.0272(-0.0006)$ & $0.0321(0.0181)$ & $0.0274(0.0030)$ & $0.0573(0.1238)$ \\
\hline & & & 3 & 26 & $0.0276(-0.0025)$ & $0.0321(0.0154)$ & $0.0277(0.0011)$ & $0.0577(0.1227)$ \\
\hline & & & 5 & 27 & $0.0269(-0.0050)$ & $0.0373(0.0439)$ & $0.0274(0.0030)$ & $0.0738(0.0698)$ \\
\hline & & & 6 & 27 & $0.0255(-0.0212)$ & $0.0427(0.0621)$ & $0.0274(0.0030)$ & $0.0679(-0.0108)$ \\
\hline & & & 6 & 25 & $0.0280(-0.0070)$ & $0.0355(0.0430)$ & $0.0282(-0.0011)$ & $0.0659(-0.0200)$ \\
\hline & & 40 & 1 & 40 & $0.0198(0.0011)$ & $0.0213(0.0096)$ & $0.0199(0.0036)$ & $0.0235(0.0150)$ \\
\hline & & & 3 & 35 & $0.0207(-0.0030)$ & $0.0234(0.0097)$ & $0.0207(-0.0005)$ & $0.0467(0.1231)$ \\
\hline & & & 4 & 37 & $0.0203(-0.0003)$ & $0.0243(0.0220)$ & $0.0203(0.0023)$ & $0.0624(0.1558)$ \\
\hline & & & 5 & 37 & $0.0204(-0.0038)$ & $0.0261(0.0366)$ & $0.0203(0.0023)$ & $0.0753(0.1531)$ \\
\hline & & & 6 & 30 & $0.0226(-0.0079)$ & $0.0266(0.0354)$ & $0.0227(-0.0035)$ & $0.0753(0.0799)$ \\
\hline & & & 11 & 30 & $0.0224(-0.0121)$ & $0.0363(0.0899)$ & $0.0227(-0.0035)$ & $0.0424(-0.1823)$ \\
\hline \multirow[t]{19}{*}{0.4} & 2.0 & 20 & 1 & 20 & $0.0509(0.0174)$ & $0.0607(0.0443)$ & $0.0516(0.0224)$ & $0.0366(0.0162)$ \\
\hline & & & 3 & 17 & $0.0513(0.0137)$ & $0.0770(0.0749)$ & $0.0528(0.0202)$ & $0.0612(0.0760)$ \\
\hline & & & 3 & 16 & $0.0529(0.0099)$ & $0.0804(0.0713)$ & $0.0543(0.0161)$ & $0.0620(0.0736)$ \\
\hline & & & 3 & 15 & $0.0546(0.0043)$ & $0.0841(0.0647)$ & $0.0558(0.0104)$ & $0.0622(0.0663)$ \\
\hline & & & 4 & 17 & $0.0507(0.0125)$ & $0.0903(0.1020)$ & $0.0528(0.0202)$ & $0.0652(0.0267)$ \\
\hline & & & 5 & 17 & $0.0508(0.0083)$ & $0.1114(0.1336)$ & $0.0528(0.0202)$ & $0.0601(-0.0540)$ \\
\hline & & & 6 & 17 & $0.0537(-0.0083)$ & $0.1458(0.1742)$ & $0.0529(0.0202)$ & $0.0503(-0.1301)$ \\
\hline & & 30 & 1 & 30 & $0.0324(0.0107)$ & $0.0374(0.0306)$ & $0.0327(0.0141)$ & $0.0301(0.0177)$ \\
\hline & & & 3 & 27 & $0.0322(0.0098)$ & $0.0418(0.0476)$ & $0.0328(0.0140)$ & $0.0539(0.1078)$ \\
\hline & & & 3 & 26 & $0.0324(0.0097)$ & $0.0421(0.0479)$ & $0.0330(0.0135)$ & $0.0541(0.1086)$ \\
\hline & & & 5 & 27 & $0.0320(0.0083)$ & $0.0509(0.0846)$ & $0.0328(0.0140)$ & $0.0713(0.0718)$ \\
\hline & & & 6 & 27 & $0.0341(0.0030)$ & $0.0581(0.1077)$ & $0.0328(0.0140)$ & $0.0687(0.0049)$ \\
\hline & & & 6 & 25 & $0.0331(0.0074)$ & $0.0652(0.1030)$ & $0.0336(0.0121)$ & $0.0681(-0.0031)$ \\
\hline & & 40 & 1 & 40 & $0.0242(0.0069)$ & $0.0273(0.0221)$ & $0.0243(0.0095)$ & $0.0253(0.0151)$ \\
\hline & & & 3 & 35 & $0.0241(0.0065)$ & $0.0292(0.0339)$ & $0.0244(0.0093)$ & $0.0447(0.1101)$ \\
\hline & & & 4 & 37 & $0.0239(0.0060)$ & $0.0312(0.0447)$ & $0.0243(0.0095)$ & $0.0591(0.1386)$ \\
\hline & & & 5 & 37 & $0.0237(0.0055)$ & $0.0335(0.0582)$ & $0.0243(0.0095)$ & $0.0703(0.1353)$ \\
\hline & & & 6 & 30 & $0.0266(0.0007)$ & $0.0475(0.0689)$ & $0.0260(0.0032)$ & $0.0763(0.0868)$ \\
\hline & & & 11 & 30 & $0.0251(-0.0064)$ & $0.0647(0.1209)$ & $0.0260(0.0033)$ & $0.0425(-0.1819)$ \\
\hline
\end{tabular}




\section{References}

Balakrishnan, N. (1985). Order statistics from the half logistic distribution, Journal of Statistical Computation and Simulation, 20, 287-309.

Balakrishnan, N. and Puthenpura, S. (1986). Best linear unbiased estimators of location and scale parameters of the half logistic distribution, Journal of Statistical Computation and Simulation, 25, 193-204.

Balakrishnan, N. and Wong, K. H. T. (1991). Approximate MLEs for the location and scale parameters of the half logistic distribution with type-II right censoring, IEEE Transactions on Reliability, 40, $140-145$.

Childs, A., Chandrasekar, B., Balakrishnan, N. and Kundu, D. (2003). Exact likelihood inference based on Type-I and Type-II hybrid censored samples from the exponential distribution, Annals of the Institute of Statistical Mathematics, 55, 319-330.

Epstein, B. (1954). Truncated life tests in the exponential case, The Annals of Mathematical Statistics, 25, 555-564.

Han, J. T. and Kang, S. B. (2008). Estimation for the double Rayleigh distribution based on multiply Type-II censored samples, Communications of the Korean Statistical Society, 15, 367-378.

Kang, S. B., Cho, Y. S. and Han, J. T. (2008). Estimation for the half logistic distribution under progressive Type-II censoring, Communications of the Korean Statistical Society, 15, 815-823. 